\title{
Erratum to: Aspirin does not increase bleeding and allogenic blood transfusion in coronary artery surgery. Thorac Cardiovasc Surg 2011;59:421-424
}

E. Hijazi ${ }^{1}$

${ }^{1}$ Division of Cardiac Surgery, Department of General Surgery, Princess Muna Al-Hussein Cardiac Center, King Abdullah University Hospital-Faculty of Medicine/Jordan University of Science and Technology, Irbid, Jordan

Address for correspondence Dr. Emad Hijazi, Department of General Surgery, King Abdullah University Hospital, Faculty of Medicine/Jordan University of Science and Technology, Irbid 22110, Jordan (e-mail: emad_hijazi@hotmail.com).

Thorac Cardiovasc Surg 2013;61:270.

ERRATUM
It has been brought to the Editor's attention that this study was not performed prospectively. The patients were in fact enrolled for surgery between June 2005 and December 2009 and assigned to one of two groups, depending on their medication. The data were then collected over time during the patients' hospital stay and finally analyzed in 2010, when approval of the local ethics committee was also obtained. This makes the study a retrospective data analysis.

Markus K. Heinemann, MD, PhD Editor-in-Chief The Thoracic and Cardiovascular Surgeon 